\title{
On the Cauchy Problem for the One-Dimensional Heat Equation
}

\author{
By F. Ginsberg
}

Abstract. In this paper we show that the Cauchy problem for the one-dimensional heat equation, though non-well posed in the sense of Hadamard, can be solved numerically. It is shown that if we admit as solutions functions for which an a priori bound is assumed in some finite rectangle in $x-t$ space then the solution depends Hölder continuously upon the given Cauchy data. The specific numerical scheme developed also exhibits the Hölder continuity so that we are sure of a meaningful numerical method.

1. Introduction. In this paper we are mainly concerned with obtaining a numerical solution to the non-well posed Cauchy problem defined by

$$
\begin{aligned}
W_{t}(x, t) & =W_{x x}(x, t) \\
W(0, t) & =\phi(t) \\
W_{x}(0, t) & =\psi(t)
\end{aligned}
$$

Where $W$ is defined in the region $R$ satisfying the inequalities

$$
0<x<1, \quad 0<t<1 .
$$

Though the problem as stated is not well posed* in the sense of Hadamard [2], we can, by the imposition of an a priori bound $B$, that is, $\mid W(x, t \mid \leqq B$ in some finite rectangle in $(x, t)$ space, show that the solutions depend Hölder continuously upon the prescribed Cauchy data. Hölder continuous dependence of the solution upon the data means: There exist constants $K, a$, and $M$ with $a$ such that $0<a \leqq 1$, for which $|W(x, t)| \leqq K$ l.u.b.0<t<1 $|\phi(t)|^{a} M^{1-a}$. In the definition we have assumed $\psi(t) \equiv 0$, for convenience, which is no loss of generality. It will be shown that the assumption $|W(x, t)| \leqq B$ in real space permits us to obtain a bound for $|W(z, t)|<M$ in $(z, t)$ space, where $z$ is the extension of $x$ into the complex domain.

The existence of a solution to the above Cauchy problem is insured by a theorem due to Holmgren [5], [8]. He has shown that a necessary and sufficient condition for the existence of a solution is that the function $g(t)$ defined by

$$
g(t)=\psi(t)+\frac{1}{\sqrt{\pi}} \int_{0}^{t} \frac{\phi^{\prime}(\tau) d \tau}{\sqrt{t-\tau}}
$$

be a function of class two [4]. Functions of class two are functions which are differentiable to any order and whose derivatives satisfy a growth condition of the type

$$
\frac{d^{n} g}{d t^{n}} \leqq \frac{M(2 n) !}{R^{2 n}}
$$

Received February 13, 1963.

* Consider $U(x, t)=e^{i k t} \cosh \sqrt{i k} x . U_{t}=U_{x x}$ is satisfied, and $U(0, t)=e^{i k t}$ and $U_{x}(0, t)=0$. Thus $|U(0, t)| \leqq 1$, but $|U(x, t)|=|\cosh \sqrt{i k} x| \sim \exp [\sqrt{2 k} x / 2]$ so the solution tends to infinity as $k$ tends to infinity for bounded $(x, t)$ domains. 
with $M$ and $R$ being two fixed positive numbers. Thus the class of analytic functions is a subset of the functions of class two.

The uniqueness of the solution to our Cauchy problem is guaranteed by the Holmgren Uniqueness Theorem for linear partial differential equations [4].

John [6] has pointed out that for practical computational purposes the Hölder continuity would be a sort of minimal requirement. That is to say, having solutions depend continuously upon the prescribed data would not lead to useful numerical methods if the continuity were only of the weak "logarithmic type" (e.g., if $u_{1}$ and $u_{2}$ are solutions to the one-dimensional heat equation with Cauchy data $f_{1}$ and $f_{2}$ such that if $\left|f_{1}-f_{2}\right| \leqq \epsilon$ then $\left|u_{1}-u_{2}\right| \leqq A /[\log 1 / \epsilon]$ where $A$ and $a$ are constants). Problems for which the numerical solutions satisfy the "Hölder condition," under the assumption of the existence of constants $K$ and $a$, are called "well-behaved".

The significance of the "well-behaved" property is that the number of significant digits in our computed solution is proportional to the number of significant digits in our data measurements. Hence, if we wish to improve the accuracy of the numerical solution we need not improve the accuracy of the measured data to an unreasonable number of significant places.

For convenience we outline our procedure. We consider the problem of generating approximate solutions for the Cauchy problem when the Cauchy data is given at some discrete set of points $\left(0, t_{i}\right)$ with a measurement error. The existence of a numerical scheme for which the approximate solution has the "Hölder property," and is thus "well-behaved," is demonstrated.

The representation

$$
W(x, t)=\sum_{n=0}^{n=\infty} \frac{x^{2 n}}{(2 n) !} \phi^{(n)}(t)
$$

which is a solution to the Cauchy problem in the special case $\psi(t)=0$, we note, can be written as

$$
W(x, t)=\sum_{k=-\infty}^{k=+\infty} C_{k} e^{i k \pi t} \cosh \sqrt{i k \pi} x .
$$

Here, $\phi(t)$ has been expanded by its Fourier series representation,

$$
\phi(t)=\sum_{k=-\infty}^{k=+\infty} C_{k} e^{i k \pi t}
$$

We then proceed to consider the case when $\phi(t)$ is known precisely on all of the interval $(0,1)$ and derive expressions which are finite representations for $\phi(t)$ (i.e. truncations) called $\phi_{N}(t)$. We show that $\phi_{N}(t)$ converges to $\phi(t)$ as $N$ tends to infinity. This, of course, means that we have to generate a method for approximating the Fourier coefficients $C_{k}$. We call the error in the computed solution, when there is no inherent data error, the truncation error. Next, if instead of knowing $\phi(t)$, and thus $\phi_{N}(t)$ precisely on $(0,1)$, we only know it at a discrete set of points $\left(0, t_{i}\right)$ with a given measurement error, we want then to represent the exact solution by means of appropriate approximation functions. The approximation scheme makes use of the Lagrange Interpolation Formulae and remainder estimates. The scheme demonstrates the importance of an appropriate choice of spacing for the data points, 
as well as the necessity of appropriately choosing the number of points at which the data is given. Minimizing the error estimates with respect to certain parameters of the approximation scheme finally leads us to the fact that the solution of the numerical problem is "well-behaved," under the assumption of an a priori bound on the solution in a rectangle in $(x, t)$ space. That is, if $W$ is the exact solution and $W_{A}$ is the approximation to this solution, then, there exist constants $\bar{C}$, and $\beta$ with $0<\beta<1$ such that $\left|W-W_{A}\right| \leqq \bar{C}^{\beta}$, where $\epsilon$ is the precision of our measurements.

A special example is presented and the numerical results obtained by the above method are compared to the solution of an equivalent boundary value problem.

The main tools used in deriving the Hölder continuity are the Sobolev lemma of functional analysis [9] and the Cauchy integral formula for analytic functions.

2. Part I. Consider the Cauchy Problem

$$
\begin{aligned}
W_{t} & =W_{x x} \\
W(0, t) & =\phi(t) \\
W(0, t) & =0
\end{aligned}
$$

where $W$ is defined in $R$ as before.

A formal solution to (1) is given by

$$
V(x, t)=\sum_{n=0}^{\infty} \frac{x^{2 n}}{(2 n) !} \phi^{(n)}(t) .
$$

We note that (2) implies

$$
\phi^{(n)}(t)=\left.\frac{\partial^{2 n} V(x, t)}{\partial x^{2 n}}\right|_{x=0} .
$$

Making use of the well known fact that the solutions to the heat equation are analytic in the space variable and assuming $|V(x, t)| \leqq B$ in $R$ one can show [3] that $|V(z, t)|<M$ in a suitable cube in $(z, t)$ space. Thus,

$$
\phi^{(n)}(t)=\left.\frac{\partial^{2 n} V(x, t)}{\partial x^{2 n}}\right|_{x=0}=\frac{(2 n) !}{2 \pi i} \oint \frac{V(\zeta, t) d}{\zeta^{2 n+1}} \leqq(2 n) ! M .
$$

We now make an additional assumption that $\phi(t)$ is such that

$$
\phi^{(n)}(0)=\phi^{(n)}(1)=0 \text { for all } n \geqq 0 .
$$

In [3] it was shown that condition (5) is no serious restriction in that all it does is to reduce the domain in which the Hölder continuity holds. This was proved by constructing a suitable $C^{\infty}$ function having the property that this function along with all its derivatives vanishes for $t=0$ and $t=1$, and on a compact subset of $t$ belonging to $(0,1)$ this function is identically 1 ([3], Chapter 4$)$.

We note that the condition $W_{x}(0, t)=0$ is also of no consequence, for it is not difficult to show that if (1) has the Hölder continuous property then so does the problem with $W_{x}(0, t)=\psi(t)$, albeit with different constants. In addition it is shown that the solutions can be continued as even functions of $x$ across the boundary $x=0$ ([3], Chapter 5$)$. 
Returning now to the function $\phi^{(n)}(t)$, define the $L_{2}$ norm of $\phi(t)$ in the usual way,

$$
\left\|\phi^{(n)}(t)\right\|^{2}=\int_{0}^{1}\left(\phi^{(n)}(t)\right)^{2} d t=\int_{0}^{1} \phi(t) \phi^{(2 n)}(t) d t=\left(\phi, \phi^{(2 n)}\right)
$$

where $(f \cdot g)$ denotes the scalar product of the functions $f$ and $g$ in the usual sense.

Using the Cauchy-Schwarz inequality we have

$$
\left\|\phi^{(n)}(t)\right\|^{2} \leqq\|\phi\|\left\|\phi^{(2 n)}\right\|
$$

where $\|\phi\|$, and $\left\|\phi^{(2 n)}\right\|$ can be estimated by using (4). From (2) we have,

$$
\mid V\left(x, t\left|\leqq \sum_{n=0}^{\infty}\right| \frac{x^{2 n}}{(2 n) !}|| \phi^{(n)}(t) \mid\right. \text {. }
$$

Now if $\phi(t)$ belongs to $C^{\infty}[0,1]$, and $\phi^{(n)}(0)=\phi^{(n)}(1)=0$ for all $n \geqq 0$, then by Sobolev's lemma

$$
\left|\phi^{(n)}(t)\right|^{2} \leqq 2\left\|\phi^{(n)}(t)\right\|^{2}+2\left\|\phi^{(n+1)}(t)\right\|^{2} .
$$

Substituting from (9) into (8) it can be shown that

$$
|V(x, t)| \leqq K\|\phi(t)\|^{1 / 2} M^{1 / 2}
$$

or

$$
|V(x, t)| \leqq K \max _{t}|\phi(t)|^{1 / 2} M^{1 / 2},
$$

with $K$ a constant. Thus (11) is a statement of the Hölder continuous dependence of the solution on the Cauchy data $\phi(t)$, valid in

$$
R^{\prime}=\left[\begin{array}{c}
0<t<1 \\
0 \leqq x \leqq \zeta<1
\end{array}\right]
$$

3. Part II. We now proceed to construct a numerical procedure which is wellbehaved. That is, we have to demonstrate the existence of a numerical scheme having the Hölder property.

Consider

$$
V(x, t)=\sum_{k=-\infty}^{k=+\infty} C_{k} e^{i k \pi t} \cosh \sqrt{i k \pi} x .
$$

It is easy to verify that (12) satisfies the Cauchy problem (1) where $\phi(t)$ has been expanded in a Fourier series on $t$ belonging to $[0,1]$

$$
\phi(t) \sim \sum_{k=-\infty}^{k=+\infty} C_{k} e^{i k \pi t}=V(0, t)
$$

Certainly, the fact that $\phi(t)$ is a function of class two, guarantees the existence of the Fourier series, where the Fourier coefficients $C_{k}$ are defined by

$$
C_{k}=\int_{0}^{1} \phi(t) e^{-i k \pi t} d t
$$


Define $V_{N}(x, t)$ by

$$
V_{N}(x, t)=\sum_{k=-N}^{k=+N} C_{k} e^{i k \pi t} \cosh \sqrt{i k \pi} x
$$

and

$$
\phi_{N}(t)=\sum_{k=-N}^{k=+N} C_{k} e^{i k \pi t}
$$

Once again it is simple to show that if $\|V(x, t)\|$ has an upper bound $M$, then $\left\|V_{N}(x, t)\right\|$ has the same upper bound.

Making use of Bessel's Inequality we have

$$
\sum_{|k| \leqq N}\left|C_{k}\right|^{2}|\cosh \sqrt{i k \pi} x|^{2} \leqq \int_{0}^{1}|V(x, t)|^{2} d t=\|V(x, t)\|^{2},
$$

( $x$ fixed). Applying Parseval's Equality,

$$
\int_{0}^{1}\left|V_{N}(x, t)\right|^{2} d t=\sum_{|k| \leqq N}\left|C_{k}\right|^{2}|\cosh \sqrt{i k \pi} x|^{2}=\left\|V_{N}(x, t)\right\|^{2} .
$$

Thus,

$$
\left\|V_{N}(x, t)\right\|^{2} \leqq\|V(x, t)\|^{2} \leqq M^{2}
$$

or

$$
\left\|V_{N}(x, t)\right\| \leqq M \text {. }
$$

Finally, applying an extension of the Sobolev Lemma [7], we have that

$$
\left|V_{N}(x, t)\right| \leqq \text { constant }\left[\max _{\rho}\left(\frac{M}{\rho^{2}}, \frac{M}{\rho}\right)\right]^{1 / 2} M^{1 / 2} .
$$

Let us assume that $\phi(t)$ is known precisely on all of $t$ belonging to [0, 1]. Applying integration by parts " $n$ " times to (14) and using (4) we have

$$
\left|C_{k}\right| \leqq \frac{(2 n) ! M}{|\pi k|^{n}}
$$

Consider now

$$
\left|\phi(t)-\phi_{N}(t)\right|=\left|\sum_{|k| \geqq N+1} C_{k} e^{i k \pi t}\right| \leqq \sum_{|k| \geqq N+1} \frac{(2 n) ! M}{(\pi k)^{n}} .
$$

Using Stirling's expansion for $n$ ! and minimizing $\frac{(2 n) !}{(\pi k)^{n}}$ with respect to $n$ for fixed $k$, we find that

$$
\sum_{|k| \geqq N+1}\left|C_{k}\right| \leqq \frac{M e}{(e-1) \exp [\sqrt{\pi(N+1)} / e]}
$$

which shows that the truncation error tends to zero as $N$ tends to infinity.

Consider now the case when $\phi(t)$ is known at some discrete set of " $s$ " points $t_{j}$ on $0<t<1$. Let us find an approximation, $\widetilde{C}_{k}$, to the actual $C_{k}$ 's, such that 
as the number of points $j$, at which we know $\phi(t)$ becomes "dense" in $(0,1)$, $\tilde{C}_{k} \rightarrow C_{k}$.

Define

$$
\tilde{C}_{k}=\int_{0}^{1} \Phi(t) e^{-i k \pi t} d t
$$

where $\Phi(t)$ is such that

$$
\Phi\left(t_{j}\right)=\phi\left(t_{j}\right), \quad j=0,1, \cdots s .
$$

Define

$$
\Phi(t)=\sum_{i} \Phi_{i}(t)
$$

where $\Phi_{i}(t)$ is a piecewise continuous function such that

$$
\Phi_{i}\left(t_{j}\right)=\left\{\begin{array}{cl}
\phi\left(t_{j}\right) & (i-1) h \leqq t \leqq i h \\
0 & i h \leqq t \leqq(i-1) h
\end{array}\right\} \quad j=0, \cdots s
$$

where $h$ is to be determined. By the Lagrange Interpolation Formula [10], a function $\Phi_{i}(t)$ interpolating to a function $\phi(t)$ in the points $t=t_{j}(j=0, \cdots s)$ is given by

$$
\Phi_{i}(t)=\sum_{k=0}^{s} \frac{\phi\left(t_{k}\right) \omega(t)}{\left(t-t_{k}\right) \omega^{\prime}\left(t_{k}\right)}
$$

where

$$
\omega(t)=\left(t-t_{0}\right) \cdots\left(t-t_{s}\right)
$$

and

$$
\omega^{\prime}\left(t_{j}\right)=\sum_{j=0}^{s} \prod_{\substack{r=0 \\ r \neq j}}^{s}\left(t_{j}-t_{r}\right)
$$

with the remainder $\left|\phi(t)-\Phi_{i}(t)\right|$ given by

$$
\left|\phi(t)-\Phi_{i}(t)\right|=\frac{\left|\phi^{(s+1)}(\xi)\right|\left|\prod_{j=0}^{s}\right|\left(t-t_{j}\right) \mid}{(s+1) !}
$$

where $\xi$ is such that $t_{0} \leqq \xi \leqq t_{s}$. Now (25) may be written as

$$
\tilde{C}_{k}=\int_{0}^{h} \Phi_{1} e^{i k \pi t} d t+\int_{h}^{2 h} \Phi_{2} e^{-i k \pi t} d t+\cdots
$$

to a finite number of terms. Consider then

$$
\left|C_{k}-\tilde{C}_{k}\right| \leqq \int_{0}^{h}\left|\phi(t)-\Phi_{1}(t)\right| d t+\int_{h}^{2 h}\left|\phi(t)-\Phi_{2}(t)\right| d t+\cdots
$$

The $i$ th integral in this sum, making use of Stirling's approximation and (4) is such that

$$
\int_{(i-1) h}^{i h}\left|\phi(t)-\Phi_{i}(t)\right| d t \leqq \frac{(2 s+2) !}{(s+1) !} M h^{s+1} .
$$


Choose $h$ now, such that

$$
h=\frac{1}{2^{3}(s+1)}
$$

therefore

$$
\int_{0}^{1}|\phi-\Phi| d t \leqq\left(\frac{1}{h}\right) \int_{(i-1) h}^{i h}\left|\phi-\Phi_{i}\right| d t=\frac{2^{3}(s+1) M}{2^{s+1}}
$$

Thus,

$$
\left|C_{k}-\tilde{C}_{k}\right| \leqq \frac{2^{3}(s+1) M}{2^{s+1}}
$$

which clearly tends to zero as $s$ tends to infinity.

Note: Although $\Phi$ is discontinuous at the end points of the subintervals $i$, this causes no problem.

We now introduce a "data" or measurement error. That is to say, instead of $\Phi_{i}\left(t_{j}\right)$ interpolating to $\phi\left(t_{j}\right)$ we consider the case when $\Phi_{i}\left(t_{j}\right)$ interpolates to $\left(\phi\left(t_{j}\right) \pm \epsilon_{i j}\right)$ with $\epsilon_{i j}$ given.

Define

$$
\hat{\Phi}(t)=\sum_{i} \hat{\Phi}_{i}(t)
$$

where

$$
\hat{\Phi}_{i}(t)=\sum_{j=0}^{s} \frac{\left(\phi\left(t_{j} \pm \epsilon_{i j}\right) \omega(t)\right.}{\left(t-t_{j}\right) \omega^{\prime}\left(t_{j}\right)}=\sum_{j=0}^{s} \frac{\phi\left(t_{j}\right) \omega(t)}{\left(t-t_{j}\right) \omega^{\prime}\left(t_{j}\right)}+\sum_{j=0}^{s} \frac{\left( \pm \epsilon_{i j}\right) \omega(t)}{\left(t-t_{j}\right) \omega^{\prime}\left(t_{j}\right)}
$$

Thus,

$$
\hat{\Phi}_{i}(t)=\Phi_{i}(t)+\sum_{j=0}^{s} \frac{\left( \pm \epsilon_{i j}\right) \omega(t)}{\left(t-t_{j}\right) \omega^{\prime}\left(t_{j}\right)} .
$$

Set

$$
\epsilon_{i}=\max _{j}\left|\epsilon_{i j}\right| \text { and } \epsilon=\max _{i} \epsilon_{i} .
$$

Proceeding in an entirely analogous manner, where now $\widetilde{\widetilde{C}}_{k}$ is the approximate Fourier coefficient, we have

$$
\begin{aligned}
\left|C_{k}-\widetilde{C}_{k}\right| \leqq \sum_{i} \int_{(i-1) h}^{i h} & \left|\phi(t)-\hat{\Phi}_{i}(t)\right| d t \\
& \leqq \sum_{i} \int_{(i-1) h}^{i h}\left|\phi(t)-\Phi_{i}(t)-\epsilon \sum_{j=0}^{\delta} \frac{\omega(t)}{\left(t-t_{j}\right) \omega^{\prime}\left(t_{j}\right)}\right| d t .
\end{aligned}
$$

By summing the accumulated error over $|k| \leqq N$, we find

$$
\sum_{|k| \leqq N}\left|C_{k}-\widetilde{\widetilde{C}}_{k}\right| \leqq(2 N+1)\left[\frac{2^{3}(s+1)}{2^{s+1}} M+\epsilon \sum_{j=0}^{s}\left|\frac{\omega(t)}{\left(t-t_{j}\right) \omega^{\prime}\left(t_{j}\right)}\right|\right] .
$$

Subdividing each of the $i$ subintervals of length $h$ by a set of equally spaced points, 
with spacing $q$, i.e.,

$$
q=\frac{h}{s+1}
$$

it can be shown that

$$
\begin{gathered}
|\omega(t)| \leqq h^{s} \\
\frac{1}{\left|\omega^{\prime}\left(t_{j}\right)\right|}<\frac{1}{(s+1) ! q^{s} \jmath !} .
\end{gathered}
$$

and finally that

$$
\sum_{j=0}^{s}\left|\frac{\omega(t)}{\left(t-t_{j}\right) \omega^{\prime}\left(t_{j}\right)}\right|=O\left(2^{s / 2}\right)
$$

Thus,

$$
\sum_{|k| \leqq N}\left|C_{k}-\widetilde{\widetilde{C}}_{k}\right| \leqq(2 N+1)\left\{\frac{2^{3} 2^{(s+1) / 2}}{2^{s+1}} M+\epsilon 2^{s}\right\} \equiv H(N, s) .
$$

Consider the function $H(N, s)$. Let us minimize the error made with respect to $s$, the number of data points for a given truncation point $N$. We find then, in some sense, the best distribution of data points for a given truncation point. Therefore $s$ is approximately given by

$$
s=\frac{\log (8 M / \epsilon)}{\log 2}
$$

and (48) can be written as:

(50) $\sum_{|k| \leqq N}\left|C_{k}-\widetilde{C}_{k}\right| \leqq(2 N+1)\left\{\frac{M \sqrt{\epsilon}}{\sqrt{8 M}}+\sqrt{8 M \epsilon}\right\}=\frac{9(2 N+1) \sqrt{8 M \epsilon}}{8}$.

We now need to show that the approximating solutions, $\widetilde{V}_{N}(x, t)$, are well-behaved, i.e., have the Hölder property.

Consider,

$$
\begin{aligned}
\left|V(x, t)-\widetilde{\widetilde{V}}_{N}(x, t)\right| & =\mid \sum_{|k| \geqq N+1} C_{k} e^{i k \pi t} \cosh \sqrt{i k \pi} x \\
& +\sum_{|k| \leqq N}\left(C_{k}-\widetilde{\widetilde{C}}_{k}\right) e^{i k \pi t} \cosh \sqrt{i k \pi} x \mid \\
\leqq & \sum_{|k| \geqq N+1}\left|C_{k}\right||\cosh \sqrt{i k \pi} x| \\
& +\sum_{|k| \leqq N}\left|C_{k}-\widetilde{\widetilde{C}}_{k}\right||\cosh \sqrt{i k \pi} x| .
\end{aligned}
$$

Applying DeMoivre's Theorem, we find an upper bound for $|\cosh \sqrt{i k \pi} x|$. To insure convergence of the sum over $|k| \geqq N+1$ we are led to the condition

$$
|x| \leqq \frac{2}{\sqrt{2} e} \text {. }
$$

By algebraic manipulations [3], it can be shown that

$$
\left|V(x, t)-\widetilde{\widetilde{V}}_{N}(x, t)\right| \leqq 4 e A e^{-\sqrt{\bar{N} / e}}+B e^{2 \sqrt{N}}
$$


where

$$
A=\frac{M e}{e-1}
$$

and

$$
B=\frac{9}{4} \sqrt{2 M \epsilon} .
$$

Minimizing the right hand side of (53) with respect to $N$, for fixed values of $\epsilon$ we find

$$
N=\frac{\left[\log \left(\frac{2 A}{B}\right)\right]^{2}}{\left(2+\frac{1}{e}\right)^{2}} .
$$

Inserting this value of $N$ in (53) we have

$$
\left|V(x, t)-\widetilde{\widetilde{V}}_{N}(x, t)\right| \leqq D M^{(4 c+1) /(4 e+2)} \epsilon^{1 /(4 e+2)}
$$

where

$$
D=\bar{K} C^{1 /(2 e+1)}\left(\frac{e}{e-1}\right)^{2 e /(2 e+1)}
$$

and where

$$
\bar{K}=\left[2^{(4 e+1)}+2^{2 e}\right]^{1 /(2 e+1)}, \text { and } C=\frac{9 \sqrt{2}}{4},
$$

which proves that the numerical scheme is well-behaved with exponent

$$
\beta=\frac{1}{4 e+2}
$$

4. Numerical Example. For convenience in the numerical example we will consider the function $\phi(t)$ as an even function of $t$. In this case we have

$$
V_{N}(x, t)=\int_{0}^{1} \phi(t) d t+4 \sum_{k=0}^{N}\left(\int_{0}^{1} \phi(t) \cos k \pi t d t\right) G(x, t, k)
$$

where

$$
\begin{aligned}
G(x, t, k)=\cosh \left(\frac{\sqrt{2 k \pi} x}{2}\right) \cos \left(\frac{\sqrt{2 k \pi} x}{2}\right) \cos k \pi t \\
\quad-\sinh \left(\frac{\sqrt{2 k \pi} x}{2}\right) \sin \left(\frac{\sqrt{2 k \pi} x}{2}\right) \sin k \pi t .
\end{aligned}
$$

We can compute $V_{N}(x, t)$ from (59) by replacing $\phi(t)$ by the appropriate Lagrange Interpolation polynomial $\hat{\Phi}(t)$. The two methods of solutions will be compared for the following problem, to find $U$ such that

$$
\begin{array}{rlr}
U_{t}(x, t) & =U_{x x}(x, t) & \\
U(1, t) & =1 & \text { for } \quad\left\{\begin{aligned}
0<x<1 \\
t>0
\end{aligned}\right\} \\
U(x, 0) & =0 &
\end{array}
$$


in the region satisfying $0<x<1$ and $t>0$. It can be verified [1] that the function

$$
V(x, t)=1+2 \sum_{n=0}^{\infty} e^{-(2 n+1)^{2} \pi^{2} t / 4} F(N, x)
$$

where

$$
F(N, x)=\left[\frac{(-1)^{n+1} 2}{(2 n+1) \pi}\right] \cos \left\{\frac{(2 n+1) \pi x}{2}\right\}
$$

is a solution to the boundary value problem defined by (60) satisfying, in addition, the condition

$$
U_{x}(0, t)=0 .
$$

The condition (62) makes this function a convenient choice against which to compare the results as computed by the method developed in this paper without too many additional manipulations.

We now return to equation (61) in order to determine the bound $M$ in complex $(z, t)$ space. By means of a simple linear transformation on the space variable $x \rightarrow x^{\prime \prime}$, and by setting

$$
t=\frac{\tau}{i}
$$

with $\tau$ a pure imaginary, we may write

$$
V\left(x^{\prime \prime}, t\right)=\frac{\pi}{2}\left[x^{\prime \prime}-2 \sum_{n=0} e^{-(n+1 / 2)^{2} \pi^{2} t} \bar{F}\left(n, x^{\prime \prime}\right)\right]
$$

where $\bar{F}\left(n, x^{\prime \prime}\right)=$

$$
\left[\sin (2 n+1) x^{\prime \prime}\right](-1)^{n} .
$$

Consider the extension into the complex plane, i.e., let $x^{\prime \prime}$ be continued into $z=x^{\prime \prime}+i y$, thus with (63) we have

$$
\begin{aligned}
V(z, \tau)=\frac{\pi}{2} \frac{d}{d z}\left[z-\vartheta_{1}(z, q)\right] & \\
= & \frac{\pi}{2}\left[1+\sum_{n=-\infty}^{n=+\infty}(-1)^{n+1}(2 n+1) e^{i\left[(n+1 / 2)^{2} \pi^{2} \tau+(2 n+1) z\right]}\right]
\end{aligned}
$$

where $\vartheta_{1}(z, q)$ is the Elliptic Theta function [11] and where we have made use of the functional equation for the Theta function [2].

Consider the doubly infinite sum, $S$, on the right hand side of (65). For convergence we require that $\operatorname{Re} f(z)<0$, where $f(z)$ is the exponent in $S$.

$$
\begin{aligned}
|S| \leqq & \sum_{n=-\infty}^{n=+\infty}(2 n+1) e^{-(n+1 / 2)^{2} \pi^{2} t-(2 n+1) y} \\
= & \sum_{n=-\infty}^{n=-1}|2 n+1| e^{-(n+1 / 2)^{2} \pi^{2} t-(2 n+1) y} \\
& +\sum_{n=1}^{n=\infty}(2 n+1) e^{-(n+1 / 2)^{2} \pi^{2} t-(2 n+1) y}+e^{-\left(\pi^{2} t / 4\right)-y}
\end{aligned}
$$


The convergence condition implies that

$$
\begin{aligned}
& \text { (a) }-y-\frac{\pi^{2} t}{4}<0 \\
& \text { (b) } \frac{-(2 n+1)^{2} \pi^{2} t}{4}-(2 n+1) y \leqq-(2 n+1) \delta(t)
\end{aligned}
$$

where $\delta(t)$ is to be specified and $\delta(t)>0$. Therefore

$$
|S| \leqq 2 \sum_{n=1}^{\infty}(2 n+1) e^{-(2 n+1) \delta(t)}+1=M
$$

where $M$ is a bound for $|V(z, t)|$ in $R$. Making use of the integral test we find

$$
\left|\sum_{n=1}^{\infty}(2 n+1) e^{-(2 n+1) \delta(t)}\right| \leqq e^{-3 \delta(t)}\left[\frac{3}{2 \delta(t)}+\frac{1}{2[\delta(t)]^{2}}\right] .
$$

If we arbitrarily choose $\delta(t)=t$, and consider $t=10^{-2}$, then we find that the bound $M$ for $V(z, t)$ in the given cube in $(z, t)$ space is

$$
M \approx 15,680 \text {. }
$$

Clearly, this is not a very sharp bound, but for our purposes it is sufficient.

Returning to the expressions for $A, B, N, s, h$ which were given previously, we find that for $M$ as given by (70) we have

$$
N \approx 20, \quad A \approx 49,548, \quad B \approx 1.24, \quad s \approx 35, \quad h=\frac{1}{2} \frac{1}{88} .
$$

The values used for $\left(\phi\left(t_{j}\right) \pm \epsilon\right)$ were the values of $V(x, t)$ for $x=0$ and $t=t_{j}$ and where $\left|\phi\left(t_{j}\right) \pm \epsilon\right| \leqq 10^{-5}$.

The values of $\widetilde{\widetilde{V}}_{20}\left(x_{i}, t_{j}\right)$ versus $V\left(x_{i}, t_{j}\right)$ and $\left|\widetilde{\widetilde{V}}_{20}\left(x_{i}, t_{j}\right)-V\left(x_{i}, t_{j}\right)\right|$ are tabulated in Table 1, where $V\left(x_{i}, t_{j}\right)$ are the values calculated from $(61)$.

5. Remarks. The value of $(1 / h)$ implies that we need to evaluate 288 integrals each containing in its interior a total of $s=35$ points, then we need to sum this over $N=20$ terms and evaluate the result over a specified grid. The enormous number of calculations involved makes imperative the use of a digital computer such as the IBM 7090 on which this problem was programmed. In spite of the speed of this machine it was estimated that to evaluate this number of integrals at so many points, making use of Simpson's Rule with an accuracy of $10^{-3}$, would require approximately 180 minutes of running time. In order to conserve time on the 7090 the total number of integrals used was 32 , with each integral containing 7 data points in its interior. The time for such a computation was approximately 4 minutes. Some typical numerical results are tabulated in the accompanying Table 1 .

We note that in the main body of this paper we have assumed that the function $\phi(t)$ is a function of class two and on this basis we found that we needed to restrict our $x$ domain to values such that $|x|<\frac{2}{\sqrt{2 e}}$. In observing the tabulated data, the results are valid for a much larger range of values of $x$. This is due to the fact that the function $\phi(t)$, in the case actually computed, satisfies a somewhat sharper upper bound than the bound $(2 n) ! M$ and thus in the computation of the truncation error: $\sum_{|k| \geqq N+1}$, we need not be as restrictive with respect to $x$ to insure convergence to zero. 


\begin{tabular}{|c|c|c|c|c|}
\hline$T$ & $X$ & $V(X, T, 20)$ & $V(X, T)$ & $\begin{array}{r}\mid V(X, T, 20) \\
-V(X, T) \mid\end{array}$ \\
\hline 0.050 & $\begin{array}{l}0.050 \\
0.100 \\
0.150 \\
0.200 \\
0.250 \\
0.300 \\
0.350 \\
0.400 \\
0.450 \\
0.500 \\
0.550 \\
0.600 \\
0.650 \\
0.700 \\
0.750 \\
0.800 \\
0.850 \\
0.900 \\
0.950 \\
1.000\end{array}$ & $\begin{array}{l}0.003848 \\
0.005246 \\
0.007802 \\
0.011864 \\
0.017946 \\
0.026739 \\
0.039131 \\
0.056222 \\
0.079321 \\
0.109927 \\
0.149684 \\
0.200296 \\
0.263395 \\
0.340356 \\
0.432060 \\
0.538593 \\
0.658921 \\
0.790542 \\
0.929186 \\
1.068605\end{array}$ & $\begin{array}{l}0.003562 \\
0.004931 \\
0.007466 \\
0.011560 \\
0.017783 \\
0.026896 \\
0.039852 \\
0.057789 \\
0.081995 \\
0.113848 \\
0.154730 \\
0.205904 \\
0.268382 \\
0.342782 \\
0.429195 \\
0.527089 \\
0.635256 \\
0.751830 \\
0.874367 \\
1.000000\end{array}$ & $\begin{array}{l}.000286 \\
.000315 \\
.000336 \\
.000304 \\
.000163 \\
.000157 \\
.000721 \\
.001567 \\
.002674 \\
.003921 \\
.005046 \\
.005608 \\
.003987 \\
.002426 \\
.002865 \\
.011504 \\
.023665 \\
.038712 \\
.054819 \\
.068605\end{array}$ \\
\hline
\end{tabular}

6. Acknowledgements. To Professor Fritz John of the Courant Institute, New York University, whose help made my thesis possible; to Dr. Farouk Odeh of IBM T. J. Watson Research Center, Yorktown Heights, for his helpful discussions with regard to the numerical problem presented herein; and to Mr. Herb Serenson of IBM T. J. Watson Research Center, Yorktown Heights, whose knowledge of programming and unselfish giving of his time made possible the numerical data presented in this paper.

International Business Machines Corporation

Yorktown Heights, New Heights, New York

1. H. Carslaw \& J. Jaeger, Conduction of Heat in Solids (Second Edition) Clarendon Press, Oxford, 1959.

2. R. Courant \& D. Hilbert, Methods of Mathematical Physics, v. 1 (First English Edition), Interscience Publishers, N. Y., 1953.

3. F. Ginsberg, The Cauchy Problem for the One-Dimensional Heat Equation, (Ph.D. Thesis NYU, 1962).

4. J. Hadamard, Lectures on Cauchy's Problem in Linear Partial Differential Equations, Dover Publications, N. Y., 1952.

5. E. Holmgren, "Sur l'extension de la methode d'integration de Riemann," Ark. Mat. Fys., 1904.

6. F. John, Comm. Pure Appl. Math., v. XIII, n. 4, November 1960.

7. F. Јон, Numerical Solution of Problems which are not Well Posed in The Sense of Hadamard, NYU, (unpublished).

8. F. John, Partial Differential Equations-Lecture Notes, NYU 1952-53.

9. L. Nirenberg, "On elliptic partial differential equations," Estrattodagli Annali Scuola Norm. Sup. Pisa; Serie III, v. XIII, f II, 1959. 
10. C. Pucci, "On the improperly posed Cauchy problem for parabolic equations," Symposium on the Numerical Treatment of Partial Differential Equations, Provisional International Computation Center, 1959.

11. J. Scarborough, Numerical Mathematical Analysis (Second Edition), The Johns Hopkins Press, Baltimore, 1950.

12. J. WALSH, Interpolation and Approximation; AMS Colloquium Publications, American Mathematical Society, Rhode Island, 1956.

13. E. WhitTaker \& J. Watson, Modern Analysis (Fourth Edition reprinted), Cambridge University Press, Cambridge, 1952. 